TRANSACTIONS OF THE

AMERICAN MATHEMATICAL SOCIETY

Volume 359, Number 1, January 2007, Pages $1-17$

S 0002-9947(06)03836-0

Article electronically published on August 24, 2006

\title{
BOUNDARY CASE OF EQUALITY IN OPTIMAL LOEWNER-TYPE INEQUALITIES
}

\author{
VICTOR BANGERT, CHRISTOPHER CROKE, SERGEI V. IVANOV, \\ AND MIKHAIL G. KATZ
}

\begin{abstract}
We prove certain optimal systolic inequalities for a closed Riemannian manifold $(X, \mathcal{G})$, depending on a pair of parameters, $n$ and $b$. Here $n$ is the dimension of $X$, while $b$ is its first Betti number. The proof of the inequalities involves constructing Abel-Jacobi maps from $X$ to its Jacobi torus $\mathbb{T}^{b}$, which are area-decreasing (on $b$-dimensional areas), with respect to suitable norms. These norms are the stable norm of $\mathcal{G}$, the conformally invariant norm, as well as other $L^{p}$-norms. Here we exploit $L^{p}$-minimizing differential 1-forms in cohomology classes. We characterize the case of equality in our optimal inequalities, in terms of the criticality of the lattice of deck transformations of $\mathbb{T}^{b}$, while the Abel-Jacobi map is a harmonic Riemannian submersion. That the resulting inequalities are actually nonvacuous follows from an isoperimetric inequality of Federer and Fleming, under the assumption of the nonvanishing of the homology class of the lift of the typical fiber of the Abel-Jacobi map to the maximal free abelian cover.
\end{abstract}

\section{Introduction, CONJECTURES, AND SOME RESUlts}

In the study of optimal systolic inequalities on a Riemannian manifold $(X, \mathcal{G})$, generalizing the Loewner inequality, two questions arise:

(1) Which stable systolic inequalities can be replaced by their conformal analogues?

(2) What are the values of the constants arising in the inequalities?

The definitions of the systolic invariants $\operatorname{stsys}_{1}(\mathcal{G}), \operatorname{confsys}_{1}(\mathcal{G})$, and $\operatorname{sys}_{1}(\mathcal{G})$ can be found in the survey [CK03, while the Abel-Jacobi map $\mathcal{A}_{X}$, in Li69, Gr96; cf. [BK04, (4.3)], [K04. The first such optimal inequality was proved in Gr99.

Received by the editors June 8, 2004

2000 Mathematics Subject Classification. Primary 53C23; Secondary 57N65, 52C07.

Key words and phrases. Abel-Jacobi map, conformal systole, deformation theorem, generalized degree, extremal lattice, free abelian cover, isoperimetric inequality, John ellipsoid, $L^{p}$-minimizing differential forms, Loewner inequality, perfect lattice, Riemannian submersion, stable systole, systolic inequality.

The first author was partially supported by DFG-Forschergruppe "Nonlinear Partial Differential Equations: Theoretical and Numerical Analysis".

The second author was supported by NSF grant DMS 02-02536 and the Max-Planck-Institut für Mathematik Bonn.

The third author was supported by grants CRDF RM1-2381-ST-02, RFBR 02-01-00090, and NS-1914.2003.1.

The fourth author was supported by the Israel Science Foundation (grants no. 620/00-10.0 and $84 / 03)$.

(C)2006 American Mathematical Society 
pp. 259-260] ( $c f$. [CK03, inequality (5.14)]) based on the techniques of D. Burago and the third author BI94, BI95].

Theorem 1.1 (M. Gromov). Let $X$ be a closed orientable manifold. Let $n=$ $\operatorname{dim}(X)$ and assume $n=b_{1}(X)=\operatorname{cuplength}_{\mathbb{R}}(X)$. Then every metric $\mathcal{G}$ on $X$ satisfies the following optimal inequality:

$$
\operatorname{stsys}_{1}(\mathcal{G})^{n} \leq \operatorname{deg}\left(\mathcal{A}_{X}\right)^{-1}\left(\gamma_{n}\right)^{\frac{n}{2}} \operatorname{vol}_{n}(\mathcal{G}),
$$

where $\gamma_{n}$ is the Hermite constant of (1.3), while $\mathcal{A}_{X}: X \rightarrow \mathbb{T}^{n}$ is the classifying map of the natural epimorphism $\pi_{1}(X) \rightarrow H_{1}(X, \mathbb{Z}) /$ torsion.

The following conformally invariant generalisation of Gromov's inequality (1.1) is immediate from Proposition 4.3, $c f$. inequality (3.3).

Theorem 1.2. Let $X$ be a closed orientable manifold. Let $n=\operatorname{dim}(X)$ and assume $n=b_{1}(X)=$ cuplength $_{\mathbb{R}}(X)$. Then every metric $\mathcal{G}$ on $X$ satisfies the following optimal inequality:

$$
\operatorname{confsys}_{1}(\mathcal{G})^{n} \leq \operatorname{deg}\left(\mathcal{A}_{X}\right)^{-1}\left(\gamma_{n}\right)^{\frac{n}{2}} .
$$

In connection with question 1 above, we aim for the following results. We show in Section 5 that if the $L^{p}$ norm is equal to the comass norm, then the minimizing measurable 1-form is in fact continuous and has constant pointwise norm. Then we can apply a Voronoi-type argument to prove that in an extremal situation, in fact, all cohomology classes are represented by 1 -forms of constant norm. This allows us to prove that a manifold $X$ such that $\operatorname{dim}(X)=b_{1}(X)=\operatorname{cuplength}_{\mathbb{R}}(X)$, which satisfies the boundary case of equality of (Gromov's) inequality relating the stable 1 -systole and the volume, must be a critical flat torus (Theorem 1.4). The proof passes via the conformally invariant strengthening of Gromov's inequality, namely Theorem 1.2 .

Given a Euclidean lattice $L \subset \mathbb{R}^{n}$, denote by $\lambda_{1}(L)>0$ the least length of a nonzero vector in $L$. A lattice $L$ is called critical if it realizes the supremum

$$
\sup _{L \subset \mathbb{R}^{n}} \frac{\lambda_{1}(L)^{n}}{\operatorname{vol}\left(\mathbb{R}^{n} / L\right)}=\left(\gamma_{n}\right)^{\frac{n}{2}},
$$

where $\gamma_{n}$ is the Hermite constant. Equivalently, $L$ is critical if the $L$-centered packing by balls is the densest one in dimension $n$. Recall that a critical (or, more generally, extremal) lattice is necessarily perfect and eutactic Bar57. The following two theorems are proved in Section 9 .

Theorem 1.3. The boundary case of equality in the conformally invariant inequality (1.2), occurs if and only if $X$ is a torus and the metric $\mathcal{G}$ is conformal to a flat critical metric.

Theorem 1.4. In the hypotheses of Theorem 1.1, equality in (1.1) is attained precisely when $X$ is a torus, while $\mathcal{G}$ is a flat metric whose deck transformations form a critical lattice.

In Section 2, we discuss a notion of degree of the Abel-Jacobi map when the dimension $n$ of the manifold $X$ exceeds its first Betti number $b$. Here we prove the positivity of the degree under a suitable topological hypothesis of homological nonvanishing. In Section 3, we extend Theorem 1.3 and Theorem 1.4 to a general pair $n \geq b$, and discuss the related results in the literature. In Section 4 , we define 
the norms involved, and state a key proposition used in the proof of Theorems 1.3 and 1.4. In Sections 5 and 6, we collect analytical results pertaining to closed forms that minimize an $L^{p}$-norm. This constitutes the analytical backbone of the paper. Sections 7 and 11 contain successive generalisations of a construction of [BI94, BI95]. The remaining sections are devoted to the proof of the results of Section 3 ,

\section{Notion of Degree When Dimension exCeEds Betti number}

Let $(X, \mathcal{G})$ be a closed Riemannian manifold, $b=b_{1}(X)$. We denote by $\mathcal{A}_{X}$ the Abel-Jacobi map,

$$
\mathcal{A}_{X}: X \rightarrow H_{1}(X, \mathbb{R}) / H_{1}(X, \mathbb{Z})_{\mathbb{R}} \simeq \mathbb{T}^{b}
$$

inducing (the natural) isomorphism in 1-dimensional cohomology. Let $\bar{X}$ be the maximal free abelian cover of $X$; cf. Definition 7.2. Denote by $\overline{\mathcal{A}}_{X}$ the proper map obtained as the lift of $\mathcal{A}_{X}$ to $\bar{X}$ :

$$
\overline{\mathcal{A}}_{X}: \bar{X} \rightarrow H_{1}(X, \mathbb{R}) \simeq \mathbb{R}^{b}
$$

If $\bar{X}$ is orientable, we fix an orientation for $\bar{X}$. In the case that $X$ is orientable, the following definition is due to M. Gromov [Gr83, p. 101].

Definition 2.1. Let $\left[\bar{F}_{X}\right] \in H_{n-b}(\bar{X}, R)$ denote the homology class of the regular fibers $\overline{\mathcal{A}}_{X}^{-1}(y)$ of $\overline{\mathcal{A}}_{X}$ where we take $R=\mathbb{Z}$ if $\bar{X}$ is orientable and $R=\mathbb{Z}_{2}$ otherwise. Then the geometric degree

$$
\operatorname{deg}\left(\mathcal{A}_{X}\right)
$$

of $\overline{\mathcal{A}}$ is the infimum of the $(n-b)$-volumes of all cycles representing $\left[\bar{F}_{X}\right]$.

Remark 2.2. If $\bar{X}$ is orientable, then changing the orientation of $\bar{X}$ will not change $\operatorname{deg}\left(\mathcal{A}_{X}\right)$. If $n=b$ and $X$ is orientable and connected, then $\operatorname{deg}\left(\mathcal{A}_{X}\right)$ is (the absolute value of) the topological degree of $\mathcal{A}_{X}$. If $n>b$ and $\left[\bar{F}_{X}\right] \neq 0$, then $\operatorname{deg}\left(\mathcal{A}_{X}\right)$ depends on the metric on $X$.

For our arguments the following obvious consequence of Definition 2.1 will be crucial.

Lemma 2.3. Suppose $f: X \rightarrow H_{1}(X, \mathbb{R}) / H_{1}(X, \mathbb{Z})_{\mathbb{R}}$ is homotopic to $\mathcal{A}_{X}$ and $y$ is a regular value of $f$. Then we have

$$
\operatorname{vol}_{n-b}\left(f^{-1}(y)\right) \geq \operatorname{deg}\left(\mathcal{A}_{X}\right)
$$

Proof. Let $\bar{y} \in H_{1}(X, \mathbb{R})$ be a preimage of $y$. Let $\bar{f}: \bar{X} \rightarrow H_{1}(X, \mathbb{R})$ be a lift of $f$. Then the covering projection $\bar{X} \rightarrow X$ maps the fiber $\bar{f}^{-1}(\bar{y})$ isometrically onto the fiber $f^{-1}(y)$, so that we have

$$
\operatorname{vol}_{n-b}\left(\bar{f}^{-1}(\bar{y})\right)=\operatorname{vol}_{n-b}\left(f^{-1}(y)\right) .
$$

On the other hand, $\bar{f}$ is homotopic to $\overline{\mathcal{A}}_{X}$ by a proper map, and hence the fiber $\bar{f}^{-1}(\bar{y})$ represents the class $\left[\bar{F}_{X}\right]$.

All the results discussed below are nonvacuous only if $\operatorname{deg}\left(\mathcal{A}_{X}\right)$ is positive. So it is important to know when this is the case. First note that this is a topological property.

Remark 2.4. If $\left[\bar{F}_{X}\right] \neq 0$, then $\operatorname{deg}\left(\mathcal{A}_{X}\right)>0$. 
This follows from the deformation theorem as stated in [Fe69, 4.2.9], complemented by [Fe69, $(4.2 .9)^{\nu}$ ] for the case of $\mathbb{Z}_{2}$-coefficients; see also [FF60], Wh99], and [Mo95, Chapter 5] for a helpful picture. The deformation theorem implies that for every compact Riemannian manifold $X$, there exists $\epsilon>0$ such that for every $q \in \mathbb{N}$ every $q$-cycle of $q$-volume smaller than $\epsilon$ can be deformed into the $(q-1)$ skeleton of a triangulation of $X$. Now, if $z$ is a $q$-cycle in $\bar{X}$ of $q$-volume smaller than $\epsilon$, then the projection of $z$ to $X$ can be deformed into the $(q-1)$-skeleton.

By the covering homotopy property, we can lift this deformation to the cover $\bar{X}$. We conclude that the homology class of $z$ vanishes regardless of the ring of coefficients; $c f$. [KR05, Proposition 5.3].

If $X$ is the quotient of the 3 -dimensional Heisenberg group by its subgroup of integral elements, then the fibers of $\mathcal{A}_{X}$ are homologically trivial in $X$ while $\left[\bar{F}_{X}\right] \neq 0$. This explains why one looks at the situation in $\bar{X}$ in Definition 2.1.

In analogy with the maximal cuplength ${ }_{\mathbb{R}}$ condition in Theorem 1.1. we note that $\left[\bar{F}_{X}\right] \neq 0$ if $X$ is orientable and if $\alpha_{1} \cup \ldots \cup \alpha_{b} \neq 0$ for every basis $\alpha_{1}, \ldots, \alpha_{b}$ of $H^{1}(X, \mathbb{R})$. Even if the real cup product vanishes, for a 3-manifold $X$ with Betti number 2 , the nonvanishing of the self-linking number of the typical fiber $\mathcal{A}_{X}^{-1}(p)$ of $\mathcal{A}_{X}: X \rightarrow \mathbb{T}^{2}$ is a sufficient condition for the nonvanishing of the fiber class $\left[\bar{F}_{X}\right]$; see [KL04]. Note that, if the minimizing rectifiable current in $\left[\bar{F}_{X}\right]$ happens to be nonsingular, then the work Lê93 produces explicit lower bounds for $\operatorname{deg}\left(\mathcal{A}_{X}\right)$ in terms of the injectivity radius and an upper bound on the sectional curvature.

\section{THE THEOREMS}

The following two results were proved in IK04.

Theorem 3.1. Let $X$ be a closed Riemannian manifold. Let $n=\operatorname{dim}(X)$ and $b=b_{1}(X)$, and assume $n \geq b \geq 1$. Then every metric $\mathcal{G}$ on $X$ satisfies the inequality

$$
\operatorname{deg}\left(\mathcal{A}_{X}\right) \operatorname{stsys}_{1}(\mathcal{G})^{b} \leq\left(\gamma_{b}\right)^{\frac{b}{2}} \operatorname{vol}_{n}(\mathcal{G}) .
$$

Corollary 3.2. Let $X$ be a closed orientable manifold. Let $b=b_{1}(X)$. Assume that $\operatorname{dim}(X)=b+1$, and $\left[\bar{F}_{X}\right] \neq 0$. Then every metric $\mathcal{G}$ on $X$ satisfies the following optimal inequality:

$$
\operatorname{stsys}_{1}(\mathcal{G})^{b} \operatorname{sys}_{1}(\mathcal{G}) \leq\left(\gamma_{b}\right)^{\frac{b}{2}} \operatorname{vol}_{b+1}(\mathcal{G}) .
$$

The main tool in studying the case of equality of the above inequalities is Proposition 3.5 which concerns a kind of an $L^{p}$-systole; $c f$. Definition 11.1. Let $p \geq 1$.

Definition 3.3. The norm \|\|$_{p}$ on $H_{1}(X ; \mathbb{R})$ is the dual norm to the $L^{p}$-norm \|\|$_{p}^{*}$ on $H^{1}(X ; \mathbb{R}) ; c f$. [BK03.

Remark 3.4. In general, if $h \in H_{k}(X, \mathbb{R})$, then the quantity $\|h\|_{\frac{n}{k}}$ is the supremum of the stable norms of $h$ with respect to all metrics that are conformal to $\mathcal{G}$ and have unit volume; cf. Gr83, 7.4.A]. In particular, one has

$$
\operatorname{stsys}_{k}(\mathcal{G}) \leq \operatorname{confsys}_{k}(\mathcal{G}) \operatorname{vol}_{n}(\mathcal{G})^{\frac{k}{n}} .
$$

Given a lattice $L$ equipped with a norm \|\| , denote by $\lambda_{1}(L,\|\|)$ the least norm of a nonzero vector in $L$. The following proposition generalizes Theorem 3.1. 
Proposition 3.5. Let $X$ be a closed orientable Riemannian manifold of unit volume. Let $b=b_{1}(X)$. Let $p \geq \max \{b, 2\}$. Then the following inequality is satisfied:

$$
\operatorname{deg}\left(\mathcal{A}_{X}\right) \lambda_{1}\left(H_{1}(X, \mathbb{Z})_{\mathbb{R}},\|\|_{p}\right)^{b} \leq\left(\gamma_{b}\right)^{\frac{b}{2}} .
$$

The proof of Proposition 3.5 appears in Section 13, By choosing $p=n$ in Proposition 3.5, we obtain the following generalisation of Theorem 1.2 and Theorem 3.1.

Theorem 3.6. Let $X$ be a closed orientable manifold. Let $n=\operatorname{dim}(X)$ and $b=$ $b_{1}(X)$. Then every metric $\mathcal{G}$ on $X$ satisfies the inequality

$$
\operatorname{deg}\left(\mathcal{A}_{X}\right) \operatorname{confsys}_{1}(\mathcal{G})^{b} \leq\left(\gamma_{b}\right)^{\frac{b}{2}} \operatorname{vol}_{n}(\mathcal{G})^{n-b} .
$$

Remark 3.7. In the case $b=2$, Theorem 3.6 follows by setting $p=2$, and applying the coarea formula to Lichnerowicz's harmonic map [Li69]; see Remark 12.1. This proves inequality (3.4) for the " $L^{2}$-systole", and the monotonicity in $p$ then proves (3.5). One can thus avoid in this case the extensions of the BI construction, described in Section 7 and Section 11. Thus our constructions can be viewed as generalisations, to $b \geq 3$, of the the properties of being area-decreasing (on the average) of Lichnerowicz's map in the case $b=2$.

In analogy with our Theorem 1.4, we prove the following.

Theorem 3.8. Let $X$ be a closed orientable Riemannan manifold. Assume that $\operatorname{dim}(X)>b_{1}(X)$. Suppose equality is attained in (3.5). Then

(1) all harmonic one-forms on $X$ have pointwise constant norm;

(2) $X$ admits a harmonic Riemannian submersion onto a flat b-torus whose deck group is a critical lattice in $\mathbb{R}^{b}$;

(3) the submersion is given by the Abel-Jacobi map defined via harmonic oneforms;

(4) the fibers of the submersion are minimal surfaces of constant $(n-b)$ dimensional area.

Note that Riemannian manifolds for which the Abel-Jacobi map is a harmonic Riemannian submersion onto a flat torus are studied in BK04. Harmonic Riemannian submersions to flat tori are examples of harmonic morphisms; see e.g. the monograph BaW03.

As a consequence of Theorem 3.8, we obtain the following; see some related results in [NV04, Na04].

Corollary 3.9. In the hypotheses of Corollary 3.2, equality in (3.2) is attained precisely when $X$ is a two-step nilpotent manifold with 1-dimensional center, while $\mathcal{G}$ is the metric of a Riemannian submersion with geodesic fibers of constant length.

The case $n=b+2$ is studied in [K04 and the companion paper BCIK04, which explores the closely related filling area conjecture. A different higher-dimensional generalisation of the Loewner inequality is studied in BK03, BK04. A proof of the Loewner inequality in genus 2 appears in [KS04, while the asymptotics of the optimal systolic ratio for large genus are studied in KS05. Similar asymptotics for the conformal 2-systole are studied in [Ka03. The work [KR04, KR05] provides a general framework for systolic geometry, in terms of a notion of systolic category, related to Lusternik-Schnirelmann category. An overview of systolic problems may be found in $\mathrm{Ka06}$. 


\section{StABle NORMS AND CONFORMAL NORMS}

Let $X$ and $Y$ be compact smooth manifolds. Let

$$
\varphi: X \rightarrow Y
$$

be a continuous map inducing an epimorphism in one-dimensional real homology. Given a Riemannian metric on $X$ (more generally, the structure of a locally simply connected length space), one defines the relative stable norm \|\|$_{s t / \varphi}$ on $H_{1}(Y ; \mathbb{R})$ by setting

$$
\|\alpha\|_{\mathrm{st} / \varphi}=\inf \left\{\|\beta\|_{\mathrm{st}} \mid \beta \in H_{1}(X ; \mathbb{R}), \varphi_{*}(\beta)=\alpha\right\},
$$

where \|\|$_{\text {st }}$ is the ordinary ("absolute") stable norm of $X$. The stable norm itself may be thought of as the relative stable norm defined by the Abel-Jacobi map to the torus $H_{1}(X, \mathbb{R}) / H_{1}(X, \mathbb{Z})_{\mathbb{R}}$.

Let $X^{n}$ be a compact Riemannian manifold, $V^{b}$ a vector space, and $\Gamma$ a lattice in $V$. We will identify $V$ and $H_{1}(V / \Gamma ; \mathbb{R})$. Let $\varphi: X \rightarrow V / \Gamma$ be a continuous map inducing an epimorphism of the fundamental groups. Note that there is a natural isomorphism

$$
\Gamma \simeq \pi_{1}(V / \Gamma) \simeq \pi_{1}(X) / \operatorname{ker}\left(\varphi_{*}\right) .
$$

The following proposition was proved in IK04. Denote by \|\|$_{E}$ the Euclidean norm on $V$, which is defined by the John ellipsoid ( $c f$. [Jo48]), of the relative stable norm \|\|$_{\mathrm{st} / \varphi}$.

Proposition 4.1. There exists a Lipschitz map $X \rightarrow\left(V / \Gamma,\|\|_{E}\right)$ which is homotopic to $\varphi$ and non-expanding on all b-dimensional areas, where $b=\operatorname{dim} V$.

The proof of the following statement may be found in BI94.

Lemma 4.2. Let $\left(V^{b},\|\|\right)$ be a Banach space. Let \|\|$_{E}$ be the Euclidean norm determined by the John ellipsoid of the unit ball of the norm \|\| . Then there exists a decomposition of \|\|$_{E}^{2}$ into rank-1 quadratic forms:

$$
\|\|_{E}^{2}=\sum_{i=1}^{N} \lambda_{i} L_{i}^{2}
$$

such that $N \leq \frac{b(b+1)}{2}, \lambda_{i}>0$ for all $i, \sum \lambda_{i}=b$, and $L_{i}: V \rightarrow \mathbb{R}$ are linear functions with $\left\|L_{i}\right\|^{*}=1$ where \|\|$^{*}$ is the dual norm to \|\| .

Note that the bound $N \leq \frac{b(b+1)}{2}+1$ appears in BI94, but one easily sees that it can be reduced by one.

Given an $n$-dimensional Riemannian manifold $X$, one defines a conformally invariant norm \|\|$_{\text {conf }}$ on $H_{1}(X ; \mathbb{R})$ as the dual norm to the $L^{n}$-norm \|\|$_{n}^{*}$ on $H^{1}(X ; \mathbb{R}) ; c f$. Remark 3.4. If $\operatorname{vol}(X)=1$, then we have an inequality \|\|$_{n}^{*} \leq\|\|^{*}$, where \|\|$^{*}$ is the comass norm in cohomology, dual to the stable norm in homology. Therefore dually, we have the inequality \|\|$_{\text {conf }} \geq\|\|_{\text {st }}$; cf. inequality (3.3). In particular, the volume form defined by the John ellipsoid of the conformal norm is greater than or equal to that of the stable norm.

For a continuous map $\varphi: X \rightarrow Y$ inducing an epimorphism in one-dimensional homology, one defines a relative conformal norm \|\|$_{\operatorname{conf} / \varphi}$ on $H_{1}(Y ; \mathbb{R})$ by setting

$$
\|\alpha\|_{\text {conf } / \varphi}=\inf \left\{\|\beta\|_{\text {conf }} \mid \beta \in H_{1}(X ; \mathbb{R}), \varphi_{*}(\beta)=\alpha\right\} .
$$

We present an "integral" version of Proposition 4.1 for conformal norms. 
Proposition 4.3. Let $X^{n}$ be a compact Riemannian manifold, $V^{n}$ a vector space, and let $\Gamma$ be a lattice in $V$. Let $\varphi: X \rightarrow V / \Gamma$ be a continuous map inducing an epimorphism of the fundamental groups. Let \|\|$_{E}$ denote the Euclidean norm on $V$ defined by the John ellipsoid of the relative conformal norm \|\|$_{\operatorname{conf} / \varphi}$. Then there exists a $C^{1}$ map

$$
f: X \rightarrow\left(V / \Gamma,\|\|_{E}\right)
$$

which is homotopic to $\varphi$ and satisfies

$$
\int_{X} \operatorname{Jac}(f) \leq 1
$$

In particular, if $\varphi$ has nonzero degree (or nonzero absolute degree), then we have the following upper bound for the volume of the torus $V / \Gamma$ :

$$
\operatorname{vol}\left(V / \Gamma,\|\|_{E}\right) \leq \frac{1}{\operatorname{deg}(\varphi)} .
$$

An adaptation of the main construction BI94, BI95, involved in the proof will be described in Section 7. We will then use it to prove Proposition 4.3 in Section 8 .

\section{Existence of $L^{p}$-Minimizers In COHOMOLOGY CLASSES}

In this section, we discuss the existence of a (weakly closed) $L^{p}$-form that minimizes the $L^{p}$-norm, $1<p<\infty$, in a given cohomology class, used in the proof of Proposition 4.3 in Section 8.

Although this is known, we could not find a good reference. The proof consists in a straightforward application of the direct method of the calculus of variations. Actually, C. Hamburger Ha92 even proved Hölder continuity of the minimizers.

Note that the existence of a continuous 1-form minimizing the comass, and defining a geodesic lamination, is proved in [FaS04, Theorem 1.7].

If $E \rightarrow X$ is a Euclidean vector bundle over a compact, oriented Riemannian manifold $X$, and if $1 \leq p<\infty$, let $L^{p}(E)$ denote the vector space of $L^{p}$-sections $s$ of $E$ endowed with the Banach norm

$$
|s|_{p}=\left(\int_{X}|s(x)|^{p} d \operatorname{vol}(x)\right)^{\frac{1}{p}} .
$$

If $p=\infty$, let $L^{\infty}(E)$ denote the essentially bounded sections of $E$ and let $|s|_{\infty}$ denote the essential supremum of the function $x \in X \rightarrow|s(x)|$.

In analogy with the case of functions $X \rightarrow \mathbb{R}$, we have:

Lemma 5.1. If $1 \leq p<\infty$ and $\frac{1}{p}+\frac{1}{q}=1$, then the natural map

$$
J_{p}: L^{p}(E) \rightarrow L^{q}(E)^{*},\left(J_{p}(s)\right)\left(s^{\prime}\right):=\int_{X}\left\langle s, s^{\prime}\right\rangle d \mathrm{vol}
$$

for $s \in L^{p}(E), s^{\prime} \in L^{q}(E)$ is an isometric isomorphism between $L^{p}(E)$ and the dual space $L^{q}(E)^{*}$ of the Banach space $L^{q}(E)$. For $1<p<\infty$ the Banach space $L^{p}(E)$ is reflexive.

Proof. If we add a vector bundle $E^{\prime}$ to $E$ such that $E \oplus E^{\prime} \rightarrow X$ is trivial, we can easily reduce the claim to the function case. For this case see e.g. A199, 4.12 and $6.10]$. 
In the sequel, the vector bundle $E$ will be the bundle $\Lambda^{k} X$ of alternating $k$-forms, $0 \leq k \leq \operatorname{dim} X=: n$. The Euclidean structure on $\Lambda^{k} X$ will be the one induced by the Riemannian metric on $X$. For $k \in\{0,1, n-1, n\}$ the corresponding Euclidean norm on the fibers of $\Lambda^{k} X$ coincides with the comass norm; cf. [Fe69, 1.8.1]. We assume that the total volume of $X$ is normalized to one.

Denote by $\Omega^{k} X$ the space of smooth $k$-forms on $X$. Given a cohomology class $\alpha \in H^{k}(X, \mathbb{R})$, denote by $\alpha_{\mathrm{dR}}$ the set of smooth closed $k$-forms $w \in \Omega^{k} X$ that represent $\alpha$. Then

$$
|\alpha|_{p}^{*}:=\inf \left\{|w|_{p} \mid w \in \alpha_{\mathrm{dR}}\right\}
$$

defines a norm on the finite-dimensional vector space $H^{k}(X, \mathbb{R})$. To see that one has $|\alpha|_{p}^{*}>0$ if $\alpha \neq 0$, note that there exists $\beta \in H^{n-k}(X, \mathbb{R})$ such that the cup product $\alpha \cup \beta \in H^{n}(X, \mathbb{R})$ is non-zero. This implies that

$$
\left|\int_{X} w \wedge \pi\right|=|(\alpha \cup \beta)([X])|>0
$$

whenever $w \in \alpha_{\mathrm{dR}}$ and $\pi \in \beta_{\mathrm{dR}}$. Because of the pointwise inequality

$$
|w \wedge \pi| \leq\left(\begin{array}{l}
n \\
k
\end{array}\right)^{\frac{1}{2}}|w||\pi|
$$

(cf. [Fe69, 1.7.5]), we obtain

$$
\int_{X}|w||\pi| d \operatorname{vol} \geq\left(\begin{array}{l}
n \\
k
\end{array}\right)^{-\frac{1}{2}}|(\alpha \cup \beta)([X])| .
$$

Hence the Hölder inequality implies

$$
|\alpha|_{p}^{*}|\beta|_{q}^{*} \geq\left(\begin{array}{l}
n \\
k
\end{array}\right)^{-\frac{1}{2}}|(\alpha \cup \beta)([X])|>0 .
$$

We let $\bar{\alpha}_{\mathrm{dR}}^{p} \subseteq L^{p}\left(\Lambda^{k} X\right)$ denote the closure of $\alpha_{\mathrm{dR}}$ with respect to the $L^{p}$-norm. Note that

$$
|\alpha|_{p}^{*}=\inf \left\{|w|_{p} \mid w \in \bar{\alpha}_{\mathrm{dR}}^{p}\right\} .
$$

Moreover, if $\beta \in H^{n-k}(X, \mathbb{R}), \frac{1}{p}+\frac{1}{q}=1, w \in \bar{\alpha}_{\mathrm{dR}}^{p}$ and $\pi \in \bar{\beta}_{\mathrm{dR}}^{q}$, then

$$
\int_{X} w \wedge \pi=[\alpha \cup \beta](X) .
$$

Since the cup product $\cup: H^{k}(X, \mathbb{R}) \times H^{n-k}(X, \mathbb{R}) \rightarrow \mathbb{R}$ is non-degenerate, this implies:

Lemma 5.2. Every smooth closed form in $\bar{\alpha}_{\mathrm{dR}}^{p}$ represents $\alpha$.

Next we prove that $\bar{\alpha}_{\mathrm{dR}}^{p}$ contains a unique $L^{p}$-form with minimal $p$-norm if $1<p<\infty$.

Proposition 5.3. Let $1<p<\infty$ and $k \in\{1, \ldots, \operatorname{dim} X-1\}$. For every $\alpha \in$ $H^{k}(X, \mathbb{R})$ there exists a unique $w \in \bar{\alpha}_{\mathrm{dR}}^{p}$ such that

$$
|w|_{p}=|\alpha|_{p}^{*}
$$


Proof. Let $\left(w_{i}\right)_{i \in \mathbb{N}}$ be a sequence in $\alpha_{\mathrm{dR}}$ such that $\lim _{i \rightarrow \infty}\left|w_{i}\right|_{p}=|\alpha|_{p}^{*}$. Since $L^{p}\left(\Lambda^{k} X\right)$ is a reflexive Banach space ( $c f$. Lemma 5.1), we can assume that the sequence $\left(w_{i}\right)_{i \in \mathbb{N}}$ converges weakly to some $w \in L^{p}\left(\Lambda^{k} X\right) ; c f$. [Al99, 6.9]. Since $\bar{\alpha}_{\mathrm{dR}}^{p}$ is a closed affine subspace of $L^{p}\left(\Lambda^{k} X\right)$, the Hahn-Banach theorem implies that $\bar{\alpha}_{\mathrm{dR}}^{p}$ is also weakly sequentially closed; $c f$. [Al99, 6.12]. This implies that $w \in \bar{\alpha}_{\mathrm{dR}}^{p}$. Moreover, the weak convergence of $\left(w_{i}\right)_{i \in \mathbb{N}}$ to $w$ implies that

$$
|w|_{p} \leq \lim _{i \rightarrow \infty}\left|w_{i}\right|_{p}=|\alpha|_{p}^{*}
$$

Using equality (5.2) and $w \in \bar{\alpha}_{\mathrm{dR}}^{p}$, we conclude that $|w|_{p}=|\alpha|_{p}^{*}$. The uniqueness of the minimizer $w$ follows from the fact that the equality $|w+\tilde{w}|_{p}=|w|_{p}+|\tilde{w}|_{p}$ can hold only if $w$ and $\tilde{w}$ are linearly dependent in $L^{p}\left(\Lambda^{k} X\right)$.

\section{EXISTENCE OF HARMONIC FORMS WITH CONSTANT NORM}

We continue to assume that the volume of $X$ is normalized to one. Then, as a direct consequence of the Hölder inequality, the function

$$
p \in[1, \infty] \rightarrow|\alpha|_{p}^{*}
$$

is (weakly) monotonically increasing for every class $\alpha \in H^{k}(X, \mathbb{R})$, where $0<k<$ $n=\operatorname{dim} X$.

Proposition 6.1. Let $\alpha \in H^{k}(X, \mathbb{R}), 0<k<n$. Assume there exist $p<p^{\prime}$ in $[1, \infty]$ such that $|\alpha|_{p}^{*}=|\alpha|_{p^{\prime}}^{*}$. Then the following holds:

(1) the function $q \in[1, \infty] \rightarrow|\alpha|_{q}^{*}$ is constant;

(2) the harmonic representative $\omega$ of $\alpha$ has constant (pointwise) norm;

(3) we have $|\omega|_{q}=|\alpha|_{q}^{*}$ for all $q \in[1, \infty]$.

Conversely, if $p \in[1, \infty)$ and if there exists a form $\omega \in \bar{\alpha}_{\mathrm{dR}}^{p}$ such that $|\omega|_{p}=|\alpha|_{p}^{*}$ and $\omega$ has constant norm almost everywhere, then

$$
|\omega|_{p}=|\omega|_{q}=|\alpha|_{q}^{*}
$$

for all $q \in[1, \infty]$. In particular, $\omega$ is harmonic.

Proof. Let $p<p^{\prime}$ in $[1, \infty]$ satisfy $|\alpha|_{p}^{*}=|\alpha|_{p^{\prime}}^{*}$. Since $q \mapsto|\alpha|_{q}^{*}$ is monotonic, we may assume that $1<p<p^{\prime}<\infty$. According to Proposition 5.3, there exists $\omega \in \bar{\alpha}_{\mathrm{dR}}^{p^{\prime}}$ such that $|\omega|_{p^{\prime}}=|\alpha|_{p^{\prime}}^{*}$. First we show that $\omega$ has constant norm. Since $\bar{\alpha}_{\mathrm{dR}}^{p^{\prime}} \subseteq \bar{\alpha}_{\mathrm{dR}}^{p}$, we see that

$$
|\alpha|_{p}^{*} \leq|\omega|_{p} \leq|\omega|_{p^{\prime}}=|\alpha|_{p^{\prime}}^{*} .
$$

Hence our assumption $|\alpha|_{p}^{*}=|\alpha|_{p^{\prime}}^{*}$ implies $|\omega|_{p}=|\omega|_{p^{\prime}}$. Therefore the function $x \in X \rightarrow\left|\omega_{x}\right|$ is almost everywhere constant.

Next, we prove that $\omega$ is indeed harmonic. If $p \leq 2$, this follows directly from

$$
|\alpha|_{p}^{*}=|\omega|_{p}=|\omega|_{2} \geq|\alpha|_{2}^{*} \geq|\alpha|_{p}^{*} .
$$

For general $p$, we will now show that $\omega$ is weakly closed and coclosed, i.e. that

$$
\int_{X}\left\langle\omega, d^{*} \pi\right\rangle d \mathrm{vol}=0 \quad \text { for all } \pi \in \Omega^{k+1} X,
$$

and

$$
\int_{X}\langle\omega, d \sigma\rangle d \mathrm{vol}=0 \quad \text { for all } \sigma \in \Omega^{k-1} X
$$


To prove (6.1), consider a fixed $\pi \in \Omega^{k+1} X$ and the linear functional

$$
\tilde{\omega} \in L^{p}\left(\Lambda^{k} X\right) \rightarrow \int_{X}\left\langle\tilde{\omega}, d^{*} \pi\right\rangle d \text { vol }
$$

on $L^{p}\left(\Lambda^{k} X\right)$. This functional vanishes on $\alpha_{\mathrm{dR}}$ and hence, by continuity, on $\omega \in \bar{\alpha}_{\mathrm{dR}}^{p}$.

Equation (6.2) is a consequence of the minimality of $|\omega|_{p}$ in $\bar{\alpha}_{\mathrm{dR}}^{p}$ and of the constancy of $\left|\omega_{x}\right|$. Note that we may assume that $\alpha \neq 0$ and hence $\left|\omega_{x}\right|=r>0$ for almost all $x \in X$. For every $\sigma \in \Omega^{k-1} X$ we have

$$
\begin{aligned}
0=\left.\frac{d}{d s}\right|_{s=0}\left(\int_{X}|\omega+s d \sigma|^{p} d \mathrm{vol}\right) & =p \int_{X}|\omega|^{p-2}\langle\omega, d \sigma\rangle d \mathrm{vol} \\
& =p r^{p-2} \int_{X}\langle\omega, d \sigma\rangle d \mathrm{vol} .
\end{aligned}
$$

Since $r \neq 0$ this implies $(6.2)$. Finally, we consider the Euclidean bundle

$$
\Lambda^{*} X=\bigoplus_{k=0}^{n} \Lambda^{k} X
$$

and the selfadjoint, first order linear differential operator $d+d^{*}$ acting on sections of $\Lambda^{*} X$. It is well known that the operator $d+d^{*}$ is elliptic. Hence the standard regularity theory for linear elliptic operators implies that $\omega$ is smooth and one has $\left(d+d^{*}\right)(\omega)=0$ in the strong sense. Since $\left(d+d^{*}\right)^{2}=\Delta$, we conclude that $\omega$ is harmonic. From the fact that $\omega$ is a harmonic form of constant norm, we can easily conclude that $|\alpha|_{q}^{*}$ does not depend on $q$. This is proved in [BK04, Proposition 8.1] for the case $k \in\{1, n-1\}$, and the proof carries over to the general case without any change.

Finally, we assume that $p \in[1, \infty)$, and that the form $\omega \in \bar{\alpha}_{\mathrm{dR}}^{p}$ has constant norm almost everywhere and satisfies $|\omega|_{p}=|\alpha|_{p}^{*}$. For every $p^{\prime} \in(p, \infty]$, we have $\omega \in \bar{\alpha}_{\mathrm{dR}}^{p^{\prime}}$ and

$$
|\alpha|_{p^{\prime}}^{*} \leq|\omega|_{p^{\prime}}=|\omega|_{p}=|\alpha|_{p}^{*} \leq|\alpha|_{p^{\prime}}^{*}
$$

Hence the first part of the proof shows that $|\alpha|_{q}^{*}$ does not depend on $q$. Since $|\omega|_{q}$ is independent of $q$ as well, we obtain

$$
|\alpha|_{p}^{*}=|\omega|_{p}=|\omega|_{q}=|\alpha|_{q}^{*}
$$

for all $q \in[1, \infty]$.

\section{The BI COnstruction ADAPTED TO CONFORMAL NORMS}

We now continue with the notation of Section 4, Consider the norm \|\|$_{\operatorname{conf} / \varphi}$ in $V$. We apply Lemma 4.2 to the norm \|\|$_{\operatorname{conf} / \varphi}$. This yields a decomposition

$$
\|\|_{E}^{2}=\sum_{i=1}^{N} \lambda_{i} L_{i}^{2}
$$

where $\lambda_{i}>0, \sum \lambda_{i}=n, L_{i} \in V^{*}$ and $\left\|L_{i}\right\|_{\text {conf } / \varphi}^{*}=1$. Then a linear map $L: V \rightarrow \mathbb{R}^{N}$ defined by

$$
L(x)=\left(\lambda_{1}^{1 / 2} L_{1}(x), \lambda_{2}^{1 / 2} L_{2}(x), \ldots, \lambda_{N}^{1 / 2} L_{N}(x)\right)
$$

is an isometry from $\left(V,\|\|_{E}\right)$ onto a subspace $L(V)$ of $\mathbb{R}^{N}$, equipped with the restriction of the standard coordinate metric of $\mathbb{R}^{N}$. (Note that the $L_{i}$ are not the components of $L$.) 
Proposition 7.1. For every $L_{i}$ there exists a weakly closed 1-form $\omega_{i}$ on $X$ from the pullback cohomology class $\omega_{i} \in \varphi^{*}\left(L_{i}\right)$ and

$$
\left\|\omega_{i}\right\|_{n}=\left\|L_{i}\right\|_{\mathrm{conf} / \varphi}^{*}=1
$$

Proof. The proof results from Proposition 5.3. Briefly, the issue is that the minimizing form $\omega_{i}$ will a priori only be an $L^{n}$-form, i.e. the $L^{n}$-limit of a sequence of smooth forms in the pullback class $\varphi^{*}\left(L_{i}\right)$. Then, it is a non-trivial result from nonlinear PDE that $\omega_{i}$ is Hölder. This can be found in To84, and in Ha92 for the case of forms of arbitrary degree.

Definition 7.2. Denote by $\bar{X}$ the covering space of $X$ defined by the subgroup $\operatorname{ker}\left(\varphi_{*}\right) \subset \pi_{1}(X)$. We denote the action of $\Gamma=\pi_{1}(X) / \operatorname{ker}\left(\varphi_{*}\right)$ on $\bar{X}$ by $(v, x) \mapsto$ $x+v$.

Corollary 7.3. There exists a $C^{1}$ function

$$
f_{i}: \bar{X} \rightarrow \mathbb{R} \text { such that } d f_{i}=\tilde{\omega}_{i},
$$

where $\tilde{\omega}_{i}$ is the lift of $\omega_{i}$. Furthermore, $f_{i}$ satisfies the relation

$$
f_{i}(x+v)=f_{i}(x)+L_{i}(v)
$$

for all $x \in \bar{X}$ and $v \in \Gamma$.

Proof. The proof of Proposition 7.1 implies the existence of $f_{i}$ with Hölder continuous first derivatives satisfying (7.2). Then (7.3) follows from the fact that $\omega_{i} \in \varphi^{*}\left(L_{i}\right)$.

Now we use the functions $f_{i}: \bar{X} \rightarrow \mathbb{R}$ of formula (7.2) to define a map $F: \bar{X} \rightarrow$ $\mathbb{R}^{N}$ as follows:

$$
F(x)=\left(\lambda_{1}^{1 / 2} f_{1}(x), \lambda_{2}^{1 / 2} f_{2}(x), \ldots, \lambda_{N}^{1 / 2} f_{N}(x)\right) .
$$

Observe that both the map $L$ of (7.1) and $F$ of (7.4) are $\Gamma$-equivariant with respect to the following action of $\Gamma$ on $\mathbb{R}^{N}$ :

$$
\Gamma \times \mathbb{R}^{N} \rightarrow \mathbb{R}^{N}, \quad(v, x) \mapsto x+L(v) .
$$

Now let $\operatorname{Pr}_{L(V)}: \mathbb{R}^{N} \rightarrow L(V)$ be the orthogonal projection to the image $L(V)$. Then the composition

$$
L^{-1} \circ \operatorname{Pr}_{L(V)} \circ F: \bar{X} \rightarrow V
$$

is a $\Gamma$-equivariant map covering a map $f: X \rightarrow V / \Gamma$. (Note that $f_{i}$ are not the components of $f$.) We will exploit it in Section 8 .

\section{Proof of Proposition 4.3}

The proof is similar to the one in [K04, with some crucial differences. Therefore we present the modified proof here. The main difference is that the functions $f_{i}$ in formula (7.2) were defined differently in IK04. Consider the norm \|\|$_{\operatorname{conf} / \varphi}$. We apply the construction of Section 7 to this norm. Since the projection is nonexpanding and the map $L$ is an isometry, it suffices to prove the proposition for the map $F$ of formula (7.4). 
Let $A=d F: T_{x} X \rightarrow \mathbb{R}^{N}$. Then

$$
\operatorname{trace}\left(A^{*} A\right)=\sum \lambda_{i}\left|d f_{i}\right|^{2} .
$$

By the inequality of geometric and arithmetic means, we have

$$
\begin{aligned}
\operatorname{Jac}(F)(x) & =\operatorname{det}\left(A^{*} A\right)^{1 / 2} \\
& \leq\left(\frac{1}{n} \operatorname{trace}\left(A^{*} A\right)\right)^{n / 2}
\end{aligned}
$$

Thus, the map $F: \bar{X} \rightarrow \mathbb{R}^{N}$ satisfies

$$
\operatorname{Jac}(F) \leq\left(\frac{1}{n} \sum \lambda_{i}\left|d f_{i}\right|^{2}\right)^{n / 2}
$$

Therefore,

$$
\begin{aligned}
\operatorname{Jac}(F) & \leq\left(\sum \frac{\lambda_{i}}{n}\left|\tilde{\omega}_{i}\right|^{2}\right)^{n / 2} \\
& \leq \sum \frac{\lambda_{i}}{n}\left|\tilde{\omega}_{i}\right|^{n}
\end{aligned}
$$

The last inequality follows from Jensen's inequality applied to the function $t \mapsto t^{n / 2}$, when $n \geq 2$. In the case $n=1$, there is only one term in the ellipsoid decomposition, so Jensen is not needed.

We now integrate the inequality over $X$. Note that $\int_{X}\left|\omega_{i}\right|^{n}=1$. This yields the desired inequality for the area of the corresponding map $X \rightarrow V / \Gamma$.

\section{Proof of Theorem 1.3 and Theorem 1.4}

Proof of Theorem 1.3. We apply the results of the preceding sections, to the case where the map $\varphi$ of (4.1) is taken to be the Abel-Jacobi map $\mathcal{A}_{X}$.

Note that equality in (8.1) is possible only if $F$ is conformal. The discussion of equality in (8.1) leads to the fact that equality in (1.2) implies that the map $f$ is conformal at all points where the differential does not vanish. If $n \geq 3$, then by [Fer77], the map $f$ is indeed a smooth local (conformal) diffeomorphism, hence a covering. Here we are assuming that the metric on $X$ is smooth. Hence $X$ is indeed a torus and $f$ is a conformal diffeomorphism.

If $n=2$, then $X$ is a torus, since $b_{1}(X)=n$ by hypothesis. Hence $f$ is a holomorphic map between 2-tori. It has degree one since it is homotopic to the Abel-Jacobi map. It follows that $f$ is a conformal diffeomorphism.

Proof of Theorem 1.4. We apply Proposition 6.1 together with Theorem 1.2 (which is immediate from Proposition 4.3) and an argument based on the perfection of a critical lattice similar to the proof of BK03. Proposition 10.5]. In more detail, let us normalize the metric to unit volume to fix ideas. The equality of the stable norm and the conformally invariant norm for each of the integral elements realizing $\lambda_{1}\left(H_{1}(X, \mathbb{Z})_{\mathbb{R}},\|\|\right)$ proves that the harmonic 1 -form in each of those integral classes has pointwise constant norm. Next, perfection implies that, in fact, every harmonic 1-form on $X$ has pointwise constant norm. It follows that the standard Abel-Jacobi map defined by those forms, is a Riemannian submersion onto a flat torus, without any ramification. Since the induced homomorphism of fundamental groups is surjective, the degree of the submersion is 1 , proving the theorem. 


\section{CASE $n \geq b$ AND $L^{p}$ NORMS IN HOMOLOGY}

In this section, we generalize the techniques of Section 7

Definition 10.1. Let $X^{n}$ and $Y^{b}$ be Riemannian manifolds, $n \geq b$, and $f: X \rightarrow Y$ a smooth map. For every $x \in X$, we consider the derivative $d_{x} f$, and define

$$
\operatorname{Jac}^{\perp} f(x)=\sup _{\sigma} \frac{\operatorname{vol}_{b}\left(d_{x} f(\sigma)\right)}{\operatorname{vol}_{b}(\sigma)}
$$

where $\sigma$ ranges over all $b$-dimensional parallelotopes in $T_{x} X$.

Obviously, if $x$ is a regular point of $f$, then the above supremum is attained at $\sigma$ lying in the orthogonal complement of the fiber $f^{-1}(f(x))$, and $\mathrm{Jac}^{\perp} f(x)=0$ if $x$ is not a regular point. We will use the following coarea formula. Every smooth map $f: X^{n} \rightarrow Y^{b}$ satisfies the identity

$$
\int_{X} \operatorname{Jac}^{\perp} f(x) d \operatorname{vol}_{n}(x)=\int_{Y} \operatorname{vol}_{n-b}\left(f^{-1}(y)\right) d \operatorname{vol}_{b}(y) .
$$

We present a version of Proposition 4.1 and Proposition 4.3, which allows us to prove inequality (3.5) for the conformal systole, as well as to handle the case of equality when $n \geq b$.

Proposition 10.2. Let $X^{n}$ be a closed Riemannian manifold of unit volume, and $V^{b}$ a vector space, $n \geq b$. Let $p \geq \max \{b, 2\}$. Let $\Gamma$ be lattice in $V$, and let $\varphi: X \rightarrow V / \Gamma$ be a continuous map inducing an epimorphism of the fundamental groups. Consider the associated $L^{p}$-norm \|\|$_{p}$ on $V$; cf. Definition 3.3. Denote by \|\|$_{E}$ the Euclidean norm on $V$, defined by the John ellipsoid of \|\|$_{p}$. Then the homotopy class of $\varphi$ contains a smooth map $f: X \rightarrow\left(V / \Gamma,\|\|_{E}\right)$ such that

$$
\int_{X} \operatorname{Jac}^{\perp} f \leq 1
$$

Proposition 10.3. In the hypotheses of Proposition 10.2, assume a strict inequality $p>\max \{b, 2\}$. If the equality is attained in (10.2), then the map $f$ is a harmonic Riemannian submersion.

In Section 11, we will generalize the BI construction of Section 7, and use it in Section 12 to prove the Propositions 10.2 and 10.3 .

\section{The Bi construction in the Case $n \geq b$}

Applying Lemma 4.2 to the norm \|\|$_{p}$ yields a decomposition

$$
\|\|_{E}^{2}=\sum_{i=1}^{N} \lambda_{i} L_{i}^{2},
$$

where $\lambda_{i}>0, \sum \lambda_{i}=b, L_{i} \in V^{*}$ and $\left\|L_{i}\right\|_{p}^{*}=1$. Then a linear map $L: V \rightarrow \mathbb{R}^{N}$ defined by

$$
L(x)=\left(\lambda_{1}^{1 / 2} L_{1}(x), \lambda_{2}^{1 / 2} L_{2}(x), \ldots, \lambda_{N}^{1 / 2} L_{N}(x)\right)
$$

is an isometry from $\left(V,\|\|_{E}\right)$ onto a subspace $L(V)$ of $\mathbb{R}^{N}$, equipped with the restriction of the standard coordinate metric of $\mathbb{R}^{N}$. (Note that the $L_{i}$ are not the components of $L$.) 
We identify the dual space $V^{*}$ of $V$ with $H^{1}(V / \Gamma ; \mathbb{R})$. Then, we have a pull-back $\operatorname{map} \varphi^{*}: V^{*} \rightarrow H^{1}(X ; \mathbb{R})$. For every $\alpha \in V^{*}$, we have

$$
\|\alpha\|_{p}^{*}=\left\|\varphi^{*}(\alpha)\right\|_{p}^{*}=\inf \left\{|\omega|_{p} \mid \omega \in \varphi^{*}(\alpha)_{\mathrm{dR}}\right\} .
$$

Definition 11.1. Fix a real $p>\max \{b, 2\}$ and let $\omega_{i}, i=1, \ldots, N$, denote the $L^{p}$-minimizer in the cohomology class $\varphi^{*}\left(L_{i}\right) \in H^{1}(X ; \mathbb{R})$.

Since $\operatorname{vol}(X)=1$, we have $\left|\omega_{i}\right|_{p}=\left\|L_{i}\right\|_{p}^{*}=1$. The rest of the construction is the same as in Section 7 In particular, we define a map

$$
F(x)=\left(\lambda_{1}^{1 / 2} f_{1}(x), \lambda_{2}^{1 / 2} f_{2}(x), \ldots, \lambda_{N}^{1 / 2} f_{N}(x)\right) .
$$

In conclusion, we obtain a map $\tilde{f}: \bar{X} \rightarrow V$ defined by

$$
\tilde{f}=L^{-1} \circ \operatorname{Pr}_{L(V)} \circ F
$$

where $\operatorname{Pr}_{L(V)}: \mathbb{R}^{N} \rightarrow L(V)$ is the orthogonal projection to $L(V)$. Clearly $\tilde{f}$ is $\Gamma$-equivariant, hence it is a lift of a map $f: X \rightarrow V / \Gamma$. (Note that the $f_{i}$ are not the components of $f$.) We show in Section 12 that $f$ satisfies inequality (10.2).

\section{Proof of Propositions 10.2 and 10.3}

Consider the map $f$ constructed in Section 11, Let $x \in X$. If $x$ is a regular point of $f$, we let $\Sigma_{x}=\left(\operatorname{ker} d_{x} f\right)^{\perp}$. Otherwise let $\Sigma_{x}$ be an arbitrary $d$-dimensional linear subspace of $T_{x} X$. Then

$$
\operatorname{Jac}^{\perp} f(x)=\operatorname{Jac}\left(\left.\left(d_{x} f\right)\right|_{\Sigma_{x}}\right) \leq \operatorname{Jac}\left(\left.\left(d_{x} F\right)\right|_{\Sigma_{x}}\right)
$$

since the projection in (11.2) is nonexpanding and $L$ is an isometry. Let $A$ be the linear map

$$
A=\left.\left(d_{x} F\right)\right|_{\Sigma_{x}}: \Sigma_{x} \rightarrow \mathbb{R}^{N} .
$$

Then, by (11.1),

$$
\operatorname{trace}\left(A^{*} A\right)=\left.\sum \lambda_{i}\left|\left(d_{x} f_{i}\right)\right|_{\Sigma_{x}}\right|^{2} \leq \sum \lambda_{i}\left|d_{x} f_{i}\right|^{2}=\sum \lambda_{i}\left|\omega_{i}(x)\right|^{2} .
$$

By the inequality of geometric and arithmetic means, we have

$$
\operatorname{Jac}\left(\left.\left(d_{x} F\right)\right|_{\Sigma_{x}}\right)=\operatorname{det}\left(A^{*} A\right)^{1 / 2} \leq\left(\frac{1}{b} \operatorname{trace}\left(A^{*} A\right)\right)^{b / 2} .
$$

Thus, the map $f$ satisfies

$$
\operatorname{Jac}^{\perp} f \leq\left(\sum \frac{\lambda_{i}}{b}\left|\omega_{i}\right|^{2}\right)^{b / 2} \leq\left(\sum \frac{\lambda_{i}}{b}\left|\omega_{i}\right|^{p}\right)^{b / p} .
$$

The last inequality follows from Jensen's inequality applied to the function $t \mapsto t^{p / 2}$; recall that $p>2$ and $\sum \lambda_{i}=b$. Integrating over $X$ yields

$$
\int_{X} \operatorname{Jac}^{\perp} f \leq \int_{X}\left(\sum \frac{\lambda_{i}}{b}\left|\omega_{i}\right|^{p}\right)^{b / p} \leq\left(\int_{X} \sum \frac{\lambda_{i}}{b}\left|\omega_{i}\right|^{p}\right)^{b / p}
$$

since $b / p<1$ and $\operatorname{vol}(X)=1$. Since $\left|\omega_{i}\right|_{p} \leq 1$, we have

$$
\int_{X} \sum \frac{\lambda_{i}}{b}\left|\omega_{i}\right|^{p}=\sum \frac{\lambda_{i}}{b} \int_{X}\left|\omega_{i}\right|^{p} \leq \sum \frac{\lambda_{i}}{b}=1,
$$

and the desired inequality (10.2) follows. 
Remark 12.1. Let $f: X \rightarrow H_{1}(X, \mathbb{R}) / H_{1}(X, \mathbb{Z})_{\mathbb{R}}$ be Lichnerowicz's harmonic map. Let $p=b=2$. Let $\omega_{1}, \omega_{2}$ be an orthonormal basis for the harmonic 1-forms with respect to the $L^{2}$ inner product. Then one has $\lambda_{1}=\lambda_{2}=1$, and the computations above can be simplified as follows:

$$
\int_{X} \operatorname{Jac}^{\perp} f=\int_{X}\left|\omega_{1} \wedge \omega_{2}\right| \leq \frac{1}{2} \int_{X}\left|\omega_{1}\right|^{2}+\left|\omega_{2}\right|^{2}=1,
$$

and the desired inequality (10.2) follows; $c f$. Remark 3.7 .

In the case of equality in (10.2), observe that all functions under integrals are continuous, hence all the inequalities throughout the argument turn to equalities.

Lemma 12.2. Equality in (10.2) implies that $\left|\omega_{i}\right|=1$ everywhere.

Proof. Equality in (12.4) implies that $\left|\omega_{i}\right|=\left|\omega_{j}\right|$ for all $i, j$. The equality in (12.5) implies that the function

$$
\sum_{i} \frac{\lambda_{i}}{b}\left|\omega_{i}\right|^{p}
$$

is constant. These together imply that $\left|\omega_{i}\right|$ is constant. Then equality in (12.6) implies that $\left|\omega_{i}\right|=1$.

Proof of Proposition 10.3. Since, by Lemma 12.2, we have $\left|\omega_{i}\right|=1$ everywhere and $\omega_{i}$ is an $L^{p}$-minimizer, Proposition 6.1 implies that $\omega_{i}$ is harmonic for every $i$. Hence $f$ is harmonic (and, in particular, smooth).

Equality in (12.3) is attained precisely when the map $A$ of formula (12.2) is a conformal linear map. Then equality in (12.1) implies that $\left.(d f)\right|_{\Sigma_{x}}$ is conformal, too. Finally, equality in (12.4) implies that $\mathrm{Jac}^{\perp} f=1$, thus $f$ is a Riemannian submersion.

\section{Proof of Proposition 3.5 and Theorem 3.8}

Proof of Proposition 3.5. We apply Proposition 10.2 to the Abel-Jacobi map $\mathcal{A}$ : $X \rightarrow \mathbb{T}^{b}=H_{1}(X ; \mathbb{R}) / H_{1}(X ; \mathbb{Z})_{\mathbb{R}}$. We obtain a map $f$ which is homotopic to $\mathcal{A}$ and satisfies inequality (10.2), where $\mathbb{T}^{b}$ is equipped with the flat Euclidean metric defined by the John ellipsoid of the unit ball of the norm \|\|$_{p}$, where the case $p=\infty$ corresponds to the stable norm. Then, by the coarea formula (10.1),

$$
\int_{X} \operatorname{Jac}^{\perp} f(x) d \operatorname{vol}_{n}(x)=\int_{\mathbb{T}^{b}} \operatorname{vol}_{n-b}\left(f^{-1}(y)\right) d \operatorname{vol}_{b}(y) .
$$

Observe that if $y \in \mathbb{T}^{b}$ is a regular value for $f$, then

$$
\operatorname{vol}_{n-b}\left(f^{-1}(y)\right) \geq \operatorname{deg}(\mathcal{A})
$$

as in Definition 2.1, Hence we have

$$
\operatorname{deg}(\mathcal{A}) \operatorname{vol}_{b}\left(\mathbb{T}^{b},\|\|_{E}\right) \leq \operatorname{vol}_{n}(X)
$$

by inequality (10.2). The proposition now results from the definition of the Hermite constant in formula (1.3).

Proof of Theorem 3.8. The theorem is immediate from Proposition 3.5 combined with Proposition 6.1, by the argument of the proof of Theorem 1.4 in Section 9 , Alternatively, one could argue as follows. In the case of equality, the equalities in (10.2) and (13.1) are attained. This implies the inequality (3.1), and the theorem 
follows from Lemma 12.2 characterizing the equality case in Proposition 10.2. Note that equality in (13.1) implies that $f^{-1}(y)$ is minimal.

\section{ACKNOWLEDGMENTS}

We are grateful to B. White for a helpful discussion of the material of Section 2 .

\section{REFERENCES}

[A199] H.W. Alt, Lineare Funktionalanalysis, 3, Auflage, Springer, 1999.

[Am04] B. Ammann, Dirac eigenvalue estimates on two-tori, J. Geom. Phys. 51 (2004), no. 3, 372-386. MR2079417 (2005f:53068)

[Bab04] I. Babenko, Géométrie systolique des variétés de groupe fondamental $\mathbb{Z}_{2}$, Sémin. Théor. Spectr. Géom. Grenoble, 22 (2004), 25-52.

[BaW03] I. Baird and John C. Wood, Harmonic morphisms between Riemannian manifolds, London Mathematical Society Monographs. New Series 29, The Clarendon Press, Oxford University Press, Oxford, 2003. MR2044031 (2005b:53101)

[BCIK04] V. Bangert, C. Croke, S. Ivanov, M. Katz, Filling area conjecture and ovalless real hyperelliptic surfaces, Geometric and Functional Analysis (GAFA) 15 (2005), no. 3, 577-597. See arXiv:math.DG/0405583 MR2221144

[BK03] V. Bangert and M. Katz, Stable systolic inequalities and cohomology products, Comm. Pure Appl. Math. 56 (2003), 979-997. Available at arXiv:math.DG/0204181 MR.1990484 (2004g:53047)

[BK04] V. Bangert and M. Katz, An optimal Loewner-type systolic inequality and harmonic one-forms of constant norm, Comm. Anal. Geom. 12 (2004), no. 3, 703-732. See arXiv:math.DG/0304494 MR/2128608

[Bar57] E. S. Barnes, On a theorem of Voronoi, Proc. Cambridge Philos. Soc. 53 (1957), 537-539. MR0086081(19:120g)

[BI94] D. Burago and S. Ivanov, Riemannian tori without conjugate points are flat, Geom. Funct. Anal. 4 (1994), no. 3, 259-269. MR1274115 (95h:53049)

[BI95] D. Burago and S. Ivanov, On asymptotic volume of tori, Geom. Funct. Anal. 5 (1995), no. 5, 800-808. MR 1354290 (96h:53041)

[CK03] C. Croke and M. Katz, Universal volume bounds in Riemannian manifolds, Surveys in Differential Geometry VIII, Lectures on Geometry and Topology held in honor of Calabi, Lawson, Siu, and Uhlenbeck at Harvard University, May 3-5, 2002 (S.T. Yau, ed.), Somerville, MA: International Press, 2003, pp. 109-137. See arXiv:math.DG/0302248 MR2039987 (2005d:53061)

[FaS04] A. Fathi and A. Siconolfi, Existence of $C^{1}$ critical subsolutions of the Hamilton-Jacobi equation, Invent. Math. 155 (2004), no. 2, 363-388. MR.2031431 (2004m:37114)

[Fe69] H. Federer, Geometric Measure Theory, Springer, 1969. MR0257325 (41:1976)

[Fe70] H. Federer, The singular sets of area minimizing rectifiable currents with codimension one and of area minimizing flat chains modulo two with arbitrary codimension, Bull. Amer. Math. Soc. 76 (1970), 767-771. MR0260981(41:5601)

[FF60] H. Federer and W. H. Fleming, Normal and integral currents, Ann. of Math. (2) 72 (1960), 458-520. MR0123260 (23:A588)

[Fer77] J. Ferrand, Sur la régularité des applications conformes, C.R. Acad. Sci. Paris Ser. A-B 284 (1977), no. 1, A77-A79. MR0470894 (57:10638)

[Gr83] M. Gromov, Filling Riemannian manifolds, J. Diff. Geom. 18 (1983), 1-147. MR0697984 (85h:53029)

[Gr96] M. Gromov, Systoles and intersystolic inequalities, Actes de la Table Ronde de Géométrie Différentielle (Luminy, 1992), 291-362, Sémin. Congr., vol. 1, Soc. Math. France, Paris, 1996. www.emis.de/journals/SC/1996/1/ps/smf_sem-cong_1_291-362.ps.gz MR1427763 (99a:53051)

[Gr99] M. Gromov, Metric structures for Riemannian and non-Riemannian spaces, Progr. in Mathematics 152, Birkhäuser, Boston, 1999. MR.1699320 (2000d:53065)

[Ha92] C. Hamburger, Regularity of differential forms minimizing degenerate functionals, J. reine angew. Math. 431 (1992), 7-64. MR.1179331 (93i:49049) 
[Iv02] S. Ivanov, On two-dimensional minimal fillings, St. Petersburg Math. J. 13 (2002), no. 1, 17-25. MR:1819361 (2002b:58016)

[IK04] S. Ivanov and M. Katz, Generalized degree and optimal Loewner-type inequalities, Israel J. Math. 141 (2004), 221-233. arXiv:math.DG/0405019 MR/2063034

[Jo48] F. John, Extremum problems with inequalities as subsidiary conditions, Studies and Essays Presented to R. Courant on his 60th Birthday, January 8, 1948, Interscience Publishers, Inc., New York, NY, 1948, pp. 187-204. MR0030135(10:719b)

[Ka03] M. Katz, Four-manifold systoles and surjectivity of period map, Comment. Math. Helv. 78 (2003), 772-786. arXiv:math.DG/0302306 MR2016695 (2005d:53062)

[Ka06] M. Katz, Systolic geometry and topology, Mathematical Surveys and Monographs, American Mathematical Society, Providence, RI, to appear.

[KL04] M. Katz and C. Lescop, Filling area conjecture, optimal systolic inequalities, and the fiber class in abelian covers, Geometry, Spectral Theory, Groups, and Dynamics, Contemporary Math., vol. 387, Amer. Math. Soc., Providence, RI, 2005. See arXiv:math.DG/0412011 MR2180208 (2006h:53030)

[KR04] M. Katz and Y. Rudyak, Lusternik-Schnirelmann category and systolic category of low dimensional manifolds, Communications on Pure and Applied Mathematics, to appear. See arXiv:math.DG/0410456

[KR05] M. Katz and Y. Rudyak, Bounding volume by systoles of 3-manifolds. See arXiv:math.DG/0504008

[KS04] M. Katz and S. Sabourau, Hyperelliptic surfaces are Loewner, Proc. Amer. Math. Soc. 134 (2006), no. 4, 1189-1195. Available at the site arXiv:math.DG/0407009 MR 2196056 (2006j:53054)

[KS05] M. Katz and S. Sabourau, Entropy of systolically extremal surfaces and asymptotic bounds, Ergodic Theory and Dynamical Systems 25 (2005), no. 4, 1209-1220. See arXiv:math.DG/0410312 MR2158402 (2006d:53038)

[KS06] M. Katz and S. Sabourau, An optimal systolic inequality for CAT(0) metrics in genus two, Pacific J. Math., to appear. See arXiv:math.DG/0501017

[Lê93] Vân Lê Hông, Curvature estimate for the volume growth of globally minimal submanifolds, Math. Ann. 296 (1993), no. 1, 103-118. MR1213374(94a:53095)

[Li69] A. Lichnerowicz, Applications harmoniques dans un tore, C.R. Acad. Sci., Sér. A 269 (1969), 912-916. MR0253254 (40:6469)

[Mo95] F. Morgan, Geometric measure theory. A beginner's guide. Second Edition, Academic Press, Boston, MA, 1995. MR1326605 (96c:49001)

[Na04] P.-A. Nagy, On length and product of harmonic forms in Kaehler geometry. See arXiv:math.DG/0406341

[NV04] P.-A. Nagy and C. Vernicos, The length of harmonic forms on a compact Riemannian manifold, Trans. Amer. Math. Soc. 356 (2004), 2501-2513. Available at arXiv:math.DG/0301369 MR:2048527 (2005f:58055)

[Pu52] P.M. Pu, Some inequalities in certain nonorientable Riemannian manifolds, Pacific J. Math. 2 (1952), 55-71. MR0048886 (14:87e)

[To84] P. Tolksdorf, Regularity for a more general class of quasilinear elliptic equations, J. Differential Equations 51 (1984), no. 1, 126-150. MR0727034 (85g:35047)

[Wh99] B. White, The deformation theorem for flat chains, Acta Math. 183 (1999), no. 2, 255271. MR 1738045 (2000m:49060)

Mathematisches Institut, Universität Freiburg, Eckerstrasse 1, 79104 Freiburg, GerMANY

E-mail address: bangert@mathematik.uni-freiburg.de

Department of Mathematics, University of Pennsylvania, Philadelphia, Pennsylvania 19104-6395

E-mail address: ccroke@math.upenn.edu

Steklov Mathematics Institute, Fontanka 27, RU-191011 St. Petersburg, Russia

E-mail address: svivanov@pdmi.ras.ru

Department of Mathematics, Bar Ilan University, Ramat Gan 52900, Israel

E-mail address: katzmik@math.biu.ac.il 